\title{
An Examination of the Role of Socioeconomic Status in the Relationship between Depression and Prostate Cancer Survivorship in a Population- Based Sample of Men from Atlantic Canada
}

\author{
Gabriela llie $^{\mathrm{a}, b, c}$ Robert Rutledge $^{c}$ Ellen Sweeney $^{\mathrm{d}}$ \\ aDepartment of Community Health and Epidemiology, Dalhousie University, Halifax, NS, Canada; ${ }^{b}$ Department \\ of Urology, Dalhousie University, Halifax, NS, Canada; 'Department of Radiation Oncology, Dalhousie University, \\ Halifax, NS, Canada; ${ }^{\mathrm{d} A t l a n t i c ~ P A T H, ~ D a l h o u s i e ~ U n i v e r s i t y, ~ H a l i f a x, ~ N S, ~ C a n a d a ~}$
}

\author{
Keywords \\ Prostate cancer · Cancer survivorship · Depression · Skin \\ cancer $\cdot$ Quality of life
}

\begin{abstract}
Objective: Prostate and skin cancer are among the most prevalent forms of cancer among men and have favorable survival rates compared to other, more aggressive forms of cancers. Recent studies have shown that the odds of depression among men with a lifetime history of prostate cancer are higher compared to men without a lifetime history of prostate cancer. Here we extend previous findings and examine the role of socioeconomic status in the relationship between depression and cancer survivorship status in a population-based sample of men from Atlantic Canada. Methods: A cross-sectional analysis was conducted on a subsample of 6,585 male participants aged 49-69 years from the 2009-2015 survey cycle of the Atlantic PATH study. The primary outcome was screening positive for mild, moderate or severe depression using the Patient Health Questionnaire (PHQ-9). The main predictor variable was cancer survivorship status (the presence of a lifetime history of prostate cancer, skin cancer, forms of cancer other than prostate or skin
\end{abstract}

cancer, or absence of a lifetime cancer diagnosis). Covariates included age, education, marital status, household income, province, ethnicity, comorbidity, and survivorship time. $\boldsymbol{R e}$ sults: An estimated $14.7 \%$ of men in this sample screened positive for mild, moderate or severe depression. Men with a history of prostate cancer were 2.60 ( $95 \% \mathrm{Cl}: 1.02,6.65)$ times more likely to screen positive for depression than men with a history of any other form of cancer. The odds ratios were 10.23 (95\% Cl: $2.82,37.49)$ or $4.00(95 \% \mathrm{Cl}: 1.20,13.34)$ times higher for survivors of prostate or skin cancer who reported a low household income to screen positive for depression compared to men with a history of any other form of cancer and high household income. Conclusions: These results extend current evidence of the association between prostate cancer survivorship and depression compared with men who never had a history of cancer diagnosis by indicating that this association still stands when the survivors of prostate cancer are compared to survivors of any other form of cancer, and further indicates that this association is moderated by household income. The findings highlight the importance of delivering mental health screening and support to prostate cancer survivors during the cancer journey, especially those with low household incomes.

(c) 2021 S. Karger AG, Basel 


\section{Introduction}

Mental health is a key component of cancer survivorship and there is a vital need to consider its impacts throughout the entire cancer journey [1]. Anxiety and depression are the most common psychological conditions affecting cancer patients and survivors by impacting overall quality of life, increasing risk of mortality, and negatively influencing treatment compliance, disease progression, and oncological outcomes [2-5]. Adults with cancer or a history of cancer more often experience health impairment, disability, fatigue, pain, comorbidities, limitations in daily functioning and other disabilities, an inability to work, and poorer self-rated health outcomes than those without cancer $[1,5]$.

Cancer patients and survivors may experience feelings of worry, loss of control, anger, sadness, confusion, and fear, as well as anxiety, mood disturbance, and changes in body image and in sexual and reproductive function [6]. The prevalence of anxiety and distress can vary by type of cancer, survivorship time, and sociodemographic variables such as age, gender, ethnicity, education, and household income $[5,6]$. For instance, while it is well established that household income directly impacts health outcomes across populations, including cancer survival, there has been less research focused on the association between income-related disparities and cancer survivors' quality of life [7-9]. This is particularly relevant when considering the long-term physical and psychological impact that cancer survivors experience which may directly impact their income [10]. Income constraints have been identified as a common barrier to symptom management and have a significant impact on the quality of life of survivors and their partners and caretakers [11].

While skin cancer is commonly diagnosed in Canada, this includes both melanoma and non-melanoma cases. Non-melanoma cases represent approximately $30 \%$ of all new cancer cases but are not accurately recorded as part of cancer surveillance programs and have 5-year relative survival rates of $100 \%$ for basal cell carcinoma and $95 \%$ for squamous cell carcinoma $[12,13]$. Considered without non-melanoma cases, melanoma skin cancer represents the seventh most commonly diagnosed cancer in Canada, and also has high rates of early detection, treatment and survival $[14,15]$.

In Canada, prostate cancer has very high survival rates and it is the most commonly diagnosed cancer among males, with 1 in 9 men expected to be diagnosed in their lifetime and accounting for $20 \%$ of new cancer cases [15]. While prostate cancer accounts for $10 \%$ of overall cancer deaths among men, the survival rates for men with localized cancer are very high, with $99 \%$ surviving at least 5 years after diagnosis [16], and 98\% surviving for at least 10 years [17].

The incidence and survival rates associated with prostate cancer highlight the importance of the overall quality of life and well-being of men during survivorship. We have previously established that there are increased rates of anxiety and depression among men with a history of prostate cancer compared to men without a lifetime history of a prostate cancer diagnosis [18-20]. We expand upon this work first by comparing depression outcomes among men with a lifetime history of prostate cancer diagnosis, skin cancer, or no lifetime history of cancer diagnosis with those with a diagnosis of any other type of cancer except prostate or skin cancer in a populationbased sample of men from Atlantic Canada. We then consider the role of socioeconomic status in moderating the relationship between depression and status of history of cancer diagnosis among men with prostate, skin and no history of cancer diagnosis compared to those with other forms of cancer diagnoses during their lifetime. Atlantic Canada is an ideal location to conduct this research, with both an aging population and the presence of the highest rates of cancer, including prostate cancer, in the country $[16,21]$.

\section{Subjects and Methods}

This study is based on an analytical sample of 6,585 men (aged 49-69 years, $M=58.94$ ) residing in Atlantic Canada who were surveyed over a span of 6 years (2009-2015) by the Atlantic Partnership for Tomorrow's Health (Atlantic PATH). The data from this project are part of a larger, pan-Canadian longitudinal cohort study examining the genetic, environmental and behavioral contributions to the development of cancer and chronic disease [22]. All procedures performed in this study were in accordance with the ethical standards of the institutional ethics committee (Dalhousie University; Project 2018-4462). The survey was conducted according to the ethics standards and principles of the World Medical Association Declaration of Helsinki guidelines for human studies. All participants had provided written informed consent prior to participating in the study. The participants completed a set of paper-and-pencil standardized surveys on sociodemographic characteristics, health history, and lifestyle factors, and they provided physical measures and biological samples. The recruitment and data collection procedures have been described elsewhere [21, 23].

\section{Measures}

Depression. The outcome measure was assessed using the Patient Health Questionnaire (PHQ-9). This is a self-reported 9-item questionnaire assessing behaviors over a period of 2 weeks, based 
on the Primary Care Evaluation of Mental Disorders (PRIME$\mathrm{MD})$ diagnostic instrument and 9 DSM-V criteria that measure positive screening for depression [24]. The items use a 4-point Likert scale, weighted from 0 to 3 , with responses ranging from "not at all," "several days," and "more than half the days" to "nearly every day," respectively [24-26]. Scores on the PHQ-9 can range between 0 and 27. Scores below 5 indicate good mental health, while scores between 5 and 9, 10 and 14, 15 and 19, and 20 and 27 indicate scoring positive for mild, moderate, moderately severe or severe depression symptoms, respectively. To ensure adequate cases, the scores on the PHQ-9 were binarily coded to depict the absence or presence of depression symptoms (scores $<5$ were coded 0 , while scores $\geq 5$ were coded 1 ).

Lifetime History of Cancer Diagnosis. The participants were asked to identify if they had ever been diagnosed with cancer and the type of cancer they had been diagnosed with. The types of cancer indicated included cancer of the bladder, brain, breast, colon, esophagus, kidney, and larynx; leukemia; cancer of the liver and of the lung and bronchus; non-Hodgkin's lymphoma; cancer of the pancreas, prostate, rectum, skin, stomach, thyroid, and trachea; and other cancers. Given the small cell counts on the outcome variable with most cancer types, with the exception of prostate (coded 1) and skin (coded 2) cancer, all other types of cancer were placed in one category (coded 3). It should be noted that skin cancer could not be distinguished between melanoma and non-melanoma. The absence of a lifetime history of cancer was coded 4 .

Covariates. Seven covariates were modeled to keep the design as similar as possible with that of Ilie et al. [18] to facilitate comparison. These covariates included age (39-49 or 50-69 years), relationship status (married or living with a partner; or divorced, widowed, separated, or single, never married), current province of residence (Nova Scotia, New Brunswick, Prince Edward Island, Newfoundland, and Labrador), education level (high-school or less; community college, trade or non-university certificate; undergraduate degree; or graduate degree), household income (under CAD 50,000; CAD 50,000-99,999; or CAD 100,000 and above), comorbidities (none, one, two or more), and ethnicity (Caucasian/White; or nonWhite). To ensure that our findings were not influenced by any changes in sample attributes across months of survivorship, we also controlled for survivorship time (months elapsed between the date of the first cancer diagnosis and survey completion).

\section{Statistical Analyses}

All analyses were performed using SPSS v.25. Cross-tab analyses were used to first assess the association between current depression status (binary) and history of cancer and the covariates. Logistic regression analyses were used to examine the relationship between the presence or absence of prostate, skin or other type of cancer or no cancer and depression in uncontrolled and controlled (age, relationship status, education, province of residence, household income, ethnicity, comorbidity, and survivorship time) analyses. A priori logistic regression analyses were used to assess the interaction between history of cancer diagnosis and each of the covariates and outcome (not controlled for covariates). None except the interaction with household income emerged. A second logistic regression assessed the role of this interaction in depression in a covariate-controlled analysis. All tests were one-tailed with statistical significance considered at $p<0.05$.

The missing-data rates across the depression were $32.9 \%(n=$ 2,168 missing) for depression, $37.7 \%$ ( $n=2,483$ missing) for his- tory of cancer diagnosis (prostate, skin, or other), $6.5 \%(n=425)$ for household income, $4.5 \%(n=296)$ for ethnicity, $0.4 \%(n=29)$ for education, $0.4 \%(n=25)$ for province, $0.5 \%(n=30)$ for comorbidities, and $0.4 \%(n=27)$ for marital status. No missing data were noted for age or survivorship time. Little's MCAR $\chi^{2}(234)=$ $2,479.91, p<0.001$, indicated that data for variables missing more than $5 \%$ were not missing at random. Multiple imputation (MI) through the iterative Markov chain Monte Carlo (MCMC) algorithm (fully conditional specification-FCS) was performed using SPSS v.25 in order to supplement missing data analyses and add confidence to the results obtained. The MCMC method can be used when the pattern of missing data is arbitrary. For each iteration, and for each variable in the order specified in the variable list, the FCS method fits a univariate model using all other available variables in the model as predictors, then imputes missing values for the variable being fit. This method continues until the maximum number of iterations is reached. For each of our analyses using MI, sensitivity analyses were performed to determine how the missing data affected the results. A random-number generator was used to generate a number of imputations between 33 and 100 (73 selected) as per literature recommendations [27].

\section{Sensitivity Analyses}

Sensitivity analyses were conducted in order to determine if our results depended on how the missing data were handled [28]. The results were pooled in a summary analysis following MI with the full MI data set $(n=6,585)$ and were compared with the listwise exclusion original data set analysis $(n=4,417)$ responses for the outcome of interest. The MI results are presented alongside the non-MI results below and were found comparable.

\section{Results}

An estimated $14.7 \%(n=651)$ of men screened positive for mild to severe depression. Table 1 presents the history of cancer diagnosis and sociodemographic characteristics of the sample. An estimated $25.8 \%$ of men with a history of prostate cancer, $10.1 \%$ of men with a history of skin cancer, $16.7 \%$ of men with other types of cancer, and $13.8 \%$ of men with no history of cancer screened positive for mild to severe depression.

Table 2 displays the results of the logistic regression analysis (original and MI pooled data sets) evaluating the relationship between history of cancer diagnosis and depression with covariate adjustment. Prostate cancer survivors statistically significantly had $2.60\left(\mathrm{OR}_{\mathrm{MI}}=1.89\right)$ times higher odds of screening positive for current mild to severe depression than men with a history of any other form of cancer. Men with a lifetime history of skin or no cancer diagnosis did not differ statistically significantly on depression screening from those with a history of other forms of cancer. Younger men (49-59 years old) statistically significantly had $2.13\left(\mathrm{OR}_{\mathrm{MI}}=1.96\right)$ times higher odds of screening positive for current depression than 
Table 1. Pearson $\chi^{2}$ analyses predicting depression symptoms by history of cancer diagnosis, age, household income, education, province, ethnicity, current marital status, and comorbidity among adult men residing in Atlantic Canada, aged 49+ years, between 2009 and 2015, for original data ( $n=4,417$ for depression symptoms)

\begin{tabular}{|c|c|c|}
\hline & $\begin{array}{l}\text { No depression symptoms } \\
(n=3,766 ; 85.3 \%), n, \%\end{array}$ & $\begin{array}{l}\text { Screening positive for mild, } \\
\text { moderate or severe depressive } \\
\text { symptoms }(n=651 ; 14.7 \%), n, \%\end{array}$ \\
\hline History of cancer diagnosis & $\left(n=2,118 ; \chi^{2}(3)=9.05^{*}\right)$ & \\
\hline Prostate & $46,74.2$ & $16,25.8$ \\
\hline Skin & $89,89.9$ & $10,10.1$ \\
\hline Other & $100,83.3$ & $20,16.7$ \\
\hline None & $1,583,86.2$ & $254,13.8$ \\
\hline Age & $\left(n=4,117 ; \chi^{2}(3)=27.94^{* * *}\right)$ & \\
\hline $49-59$ years & $1,921,82.6$ & $405,17.4$ \\
\hline $60-69$ years & $1,845,88.2$ & $246,11.8$ \\
\hline Household income & $\left(n=4,168 ; \chi^{2}(3)=27.72^{* * *}\right)$ & \\
\hline $\mathrm{CAD}<50,000$ & $657,79.9$ & $165,20.1$ \\
\hline CAD 50,000-99,999 & $1,509,84.8$ & $271,15.2$ \\
\hline $\mathrm{CAD} \geq 100,000$ & $1,378,88.0$ & $188,12.0$ \\
\hline Education & $\left(n=4,402 ; \chi^{2}(3)=19.27^{* * *}\right)$ & \\
\hline High school or less & $848,82.7$ & $178,17.3$ \\
\hline Community college & $1,381,83.9$ & $265,16.8$ \\
\hline Undergraduate degree & $838,87.4$ & $121,12.6$ \\
\hline Graduate degree & $685,88.8$ & $86,12.6$ \\
\hline Province & $\left(n=4,402, \chi^{2}(3)=1.60\right)$ & \\
\hline Nova Scotia & $2,499,85.1$ & $439,14.9$ \\
\hline New Brunswick & $761,86.6$ & $118,13.4$ \\
\hline Prince Edward Island & $70,86.4$ & $11,13.6$ \\
\hline Newfoundland and Labrador & $426,84.5$ & $78,15.5$ \\
\hline Ethnicity & $\left(n=4,176 ; \chi^{2}(3)=19.27^{*}\right)$ & \\
\hline Other & $179,80.3$ & $44,19.7$ \\
\hline Caucasian & $3,392,85.8$ & $561,14.2$ \\
\hline Current marital status & $\left(n=4,410 ; \chi^{2}(3)=19.94^{* * *}\right)$ & \\
\hline Divorced or single & $375,78.5$ & $103,21.5$ \\
\hline Married or with partner & $3,386,86.1$ & $546,13.9$ \\
\hline Comorbidities & $\left(n=4,407 ; \chi^{2}(3)=92.98^{* * *}\right)$ & \\
\hline None & $2,128,89.0$ & $264,11.0$ \\
\hline One & $1,154,84.0$ & $220,16.0$ \\
\hline Two or more & $474,73.9$ & $641,26.1$ \\
\hline Survivorship time & $\left(n=4,417 ; \chi^{2}(53)=87.55^{* *}\right)$ & \\
\hline $\begin{array}{l}\text { Number of months since diagnosis } \\
\quad(0-69 \text { months), } n, M \text { (SD) }\end{array}$ & $3,766,55.97(13.20)$ & $651,53.32(13.38)$ \\
\hline
\end{tabular}

older men (60-69 years old). Men with a household income lower than CAD 50,000 a year and those with a household income of CAD 50,000-99,000 a year statistically significantly had $2.21\left(\mathrm{OR}_{\mathrm{MI}}=1.62\right)$ and 1.70 $\left(\mathrm{OR}_{\mathrm{MI}}=1.31\right)$ times higher odds for screening positive for depression, respectively, than men presenting with a higher household income. Men with one comorbidity and those with two or more comorbidities also had 1.62 $\left(\mathrm{OR}_{\mathrm{MI}}=1.61\right)$ and $2.63\left(\mathrm{OR}_{\mathrm{MI}}=2.99\right)$ times higher odds of screening positive for depression than those without comorbidities. Lastly, men who identified as being divorced, widowed, separated, or single/never married statistically significantly had $1.63\left(\mathrm{OR}_{\mathrm{MI}}=1.34\right)$ times higher odds for screening positive for depression than men who identified as being married or currently in a relationship. Education, province, ethnicity, and survivorship time since diagnosis did not contribute significantly to the model. 
Table 2. Binary logistic regression analysis predicting depression symptoms by the history of cancer and covariates among adult men residing in Atlantic Canada, aged 49+ years, between 2009 and 2015, for original data $(n=$ 4,168 depression symptoms) and multiple imputation pooled data ( $n_{\mathrm{MI}}=6,585$ for depression symptoms)

\begin{tabular}{|c|c|c|}
\hline & \multicolumn{2}{|c|}{$\begin{array}{l}\text { Screening positive for mild, moderate or severe depression } \\
\text { symptoms (reference: no depression symptoms) }\end{array}$} \\
\hline & $\begin{array}{l}n=4,168^{\mathrm{a}} \\
\text { OR }(95 \% \mathrm{CI})\end{array}$ & $\begin{array}{l}n=6,585^{\mathrm{b}} \\
\mathrm{OR}_{\mathrm{MI}}(95 \% \mathrm{CI})\end{array}$ \\
\hline History of cancer diagnosis & \multicolumn{2}{|c|}{$\chi^{2}(17)=81.58^{* * *}$} \\
\hline Prostate & $2.60(1.02,6.65)^{*}$ & $1.89(1.09,3.26)^{*}$ \\
\hline Skin & $0.73(0.28,1.91)$ & $0.69(0.29,1.66)$ \\
\hline No cancer & $0.89(0.29,2.75)$ & $1.14(0.38,3.38)$ \\
\hline Other cancer types & 1.0 reference & 1.0 reference \\
\hline Household income (annual) & \multicolumn{2}{|c|}{$\chi^{2}(6)=14.61^{* *}$} \\
\hline $\mathrm{CAD}<50,000$ & $2.21(1.44,3.39)^{* * *}$ & $1.62(1.23,2.14)^{* *}$ \\
\hline CAD 50,000-99,999 & $1.70(1.21,2.39)^{* *}$ & $1.31(1.04,1.64)^{*}$ \\
\hline $\mathrm{CAD} \geq 100,000$ & 1.0 reference & 1.0 reference \\
\hline Age & \multicolumn{2}{|c|}{$\chi^{2}(1)=17.28^{* * *}$} \\
\hline $49-59$ years & $2.13(1.49,3.04)^{* * *}$ & $1.96(1.52,2.52)^{* *}$ \\
\hline $60-69$ years & 1.0 reference & 1.0 reference \\
\hline Education & \multicolumn{2}{|c|}{$\chi^{2}(3)=2.96$} \\
\hline High school or less & $0.98(0.60,1.59)$ & $1.34(0.99,1.81)$ \\
\hline Community college & $0.86(0.56,1.31)$ & $1.24(0.94,1.62)$ \\
\hline Undergraduate degree & $0.70(0.43,1.14)$ & $1.05(0.78,1.42)$ \\
\hline Graduate degree & \multirow{2}{*}{\multicolumn{2}{|c|}{$\chi^{2}(3)=0.51$}} \\
\hline Province & & \\
\hline Nova Scotia & $1.11(0.76,1.63)$ & $1.20(0.90,1.59)$ \\
\hline New Brunswick & $1.01(0.70,1.45)$ & $0.93(0.68,1.28)$ \\
\hline Prince Edward Island & $0.94(0.43,2.43)$ & $0.99(0.48,2.04)$ \\
\hline Newfoundland and Labrador & 1.0 reference & 1.0 reference \\
\hline Ethnicity & \multicolumn{2}{|c|}{$\chi^{2}(1)=0.15$} \\
\hline Other & $1.11(0.65,1.90)$ & $1.44(1.00,2.07)$ \\
\hline Caucasian & 1.0 reference & 1.0 reference \\
\hline Current marital status & \multicolumn{2}{|c|}{$\chi^{2}(1)=5.22^{*}$} \\
\hline Divorced or single & $1.63(1.07,2.48)^{*}$ & $1.34(1.04,1.74)^{*}$ \\
\hline Married or with partner & 1.0 reference & 1.0 reference \\
\hline Comorbidities & \multicolumn{2}{|c|}{$\chi^{2}(2)=28.05^{* * *}$} \\
\hline One & $1.62(1.19,2.22)^{* *}$ & $1.61(1.32,1.96)^{* * *}$ \\
\hline Two or more & $2.63(1.82,3.78)^{* * *}$ & $2.99(2.37,3.75)^{* * *}$ \\
\hline None & \multirow{2}{*}{\multicolumn{2}{|c|}{$\chi^{2}(1)=0.23$}} \\
\hline Survivorship time & & \\
\hline Number of months since diagnosis & $1.00(0.98,1.03)$ & $0.99(0.97,1.01)$ \\
\hline
\end{tabular}

*** $p<0.001,{ }^{* *} p<0.01,{ }^{*} p<0.05$, two-tailed test. ${ }^{\text {a }}$ Original data. ${ }^{\mathrm{b}}$ Multiple imputation pooled data based on 73 imputations.

The interaction terms between the predictor and the covariates who were identified to have a statistically significant association with depression screening status (age, household income, multimorbidity, and marital status; Table 2) were each evaluated in a binary logistic regression predicting depression, and were found to be not sta- tistically significantly associated with the outcome $(p>$ $0.05)$, except for household income $\left(\chi^{2}(1)=22.8, p<\right.$ 0.001 ). Table 3 presents the results of the binary logistic regression evaluating the interaction term between history of cancer diagnosis and household income while controlling for the covariates. Men who identified as hav- 
Table 3. Binary logistic regression analysis predicting depression symptoms by the interaction between history of cancer and household income, and covariates among adult men residing in Atlantic Canada, aged 49+ years, between 2009 and 2015, for original data ( $n=4,168$ depression symptoms) and multiple imputation pooled data $\left(n_{\mathrm{MI}}=6,585\right.$ for depression symptoms)

\begin{tabular}{|c|c|c|}
\hline & \multicolumn{2}{|c|}{$\begin{array}{l}\text { Screening positive for mild, moderate or } \\
\text { severe depression symptoms (reference: no } \\
\text { depression symptoms) }\end{array}$} \\
\hline & $\begin{array}{l}n=4,168^{\mathrm{a}} \\
\text { OR }(95 \% \mathrm{CI})\end{array}$ & $\begin{array}{l}n=6,585^{\mathrm{b}} \\
\mathrm{OR}_{\mathrm{MI}}(95 \% \mathrm{CI})\end{array}$ \\
\hline Type of history of cancer diagnosis $\times$ annual household income & \multicolumn{2}{|c|}{$\chi^{2}(18)=91.47^{* * *}$} \\
\hline Prostate $\times$ CAD $<50,000$ & $10.23(2.82,37.49)^{* *}$ & $6.86(2.01,23.39)^{* *}$ \\
\hline Prostate $\times$ CAD 50,000-99,999 & $1.53(0.39,6.02)$ & $1.70(0.60,4.81)$ \\
\hline Skin $\times$ CAD $<50,000$ & $4.00(1.20,13.34)^{*}$ & $2.35(0.86,6.36)$ \\
\hline Skin $\times$ CAD 50,000-99,999 & $0.43(0.10,1.96)$ & $0.41(0.10,1.73)$ \\
\hline No cancer $\times$ CAD $<50,000$ & $2.02(1.27,3.20)^{* *}$ & $1.54(1.16,2.06)^{* *}$ \\
\hline No cancer $\times$ CAD 50,000-99,999 & $1.87(1.32,2.66)^{* * *}$ & $1.33(1.07,1.68)^{*}$ \\
\hline Other cancer $\times \mathrm{CAD} \geq 100,000$ & 1.0 reference & 1.0 reference \\
\hline Age & \multicolumn{2}{|c|}{$\chi^{2}(1)=18.33^{* * *}$} \\
\hline $49-59$ years & $1.98(1.45,2.70)^{* * *}$ & $1.93(1.59,2.34)^{* * *}$ \\
\hline $60-69$ years & 1.0 reference & 1.0 reference \\
\hline Education & \multicolumn{2}{|c|}{$\chi^{2}(3)=4.06$} \\
\hline High school or less & $0.95(0.59,1.53)$ & $1.32(0.98,1.78)$ \\
\hline Community college & $0.83(0.54,1.28)$ & $1.24(0.94,1.64)$ \\
\hline Undergraduate degree & $0.94(0.43,2.05)$ & $1.02(0.76,1.39)$ \\
\hline Graduate degree & 1.0 reference & 1.0 reference \\
\hline Province & \multicolumn{2}{|c|}{$\chi^{2}(3)=0.56$} \\
\hline Nova Scotia & $1.10(0.75,1.62)$ & $1.19(0.90,1.57)$ \\
\hline New Brunswick & $0.99(0.69,1.43)$ & $0.92(0.67,1.27)$ \\
\hline Prince Edward Island & $0.94(0.43,2.05)$ & $0.93(0.46,1.94)$ \\
\hline Newfoundland and Labrador & 1.0 reference & 1.0 reference \\
\hline Ethnicity & \multicolumn{2}{|c|}{$\chi^{2}(1)=0.31$} \\
\hline Other & $1.16(0.68,1.99)$ & $1.43(1.00,2.04)$ \\
\hline Caucasian & 1.0 reference & 1.0 reference \\
\hline Current marital status & \multicolumn{2}{|c|}{$\chi^{2}(1)=4.87^{*}$} \\
\hline Divorced or single & $1.61(1.06,2.46)^{*}$ & $1.35(1.05,1.75)^{*}$ \\
\hline Married or with partner & 1.0 reference & 1.0 reference \\
\hline Comorbidities & \multicolumn{2}{|c|}{$\chi^{2}(2)=28.74^{* * *}$} \\
\hline One & $1.60(1.17,2.20)^{* *}$ & $1.60(1.31,1.94)^{* * *}$ \\
\hline Two or more & $2.67(1.85,3.84)^{* * *}$ & $2.96(2.36,3.72)^{* * *}$ \\
\hline None & 1.0 reference & 1.0 reference \\
\hline Survivorship time & \multicolumn{2}{|c|}{$\chi^{2}(1)=0.27$} \\
\hline Number of months since diagnosis & $1.00(0.99,1.01)$ & $0.99(0.98,1.00)$ \\
\hline
\end{tabular}

ing had a history of prostate cancer diagnosis and a household income below CAD 50,000 a year statistically significantly had odds that were $10.23\left(\mathrm{OR}_{\mathrm{MI}}=6.86\right)$ and $4.00\left(\mathrm{OR}_{\mathrm{MI}}=2.35, \mathrm{~ns}\right.$. $)$ higher for screening positive for current depression than men with a history of other forms of cancer diagnosis and those with an annual household income of CAD 100,000 or more, respectively. Men without a lifetime history of cancer who identified as currently having a low (below CAD 50,000) or a moderate household income (CAD 50,000-99,999) annually also statistically significantly had $2.02\left(\mathrm{OR}_{\mathrm{MI}}=1.54\right)$ or 1.87 $\left(\mathrm{OR}_{\mathrm{MI}}=1.33\right)$ times higher odds of screening positive for 
current depression, respectively, than men without a history of cancer diagnosis and an annual household income of CAD 100,000 or more. Men of younger age and those who identified as being divorced, widowed, separated, or single/never married statistically significantly had 1.98 $\left(\mathrm{OR}_{\mathrm{MI}}=1.93\right)$ and $1.61\left(\mathrm{OR}_{\mathrm{MI}}=1.35\right)$ times higher odds for screening positive for depression, respectively, than men who identified as being of older age and those who were married/in a relationship, respectively. Men with one comorbidity and those with two or more comorbidities also had $1.60\left(\mathrm{OR}_{\mathrm{MI}}=1.60\right)$ and $2.67\left(\mathrm{OR}_{\mathrm{MI}}=2.96\right)$ times higher odds of screening positive for depression than those without comorbidities. Education, province, ethnicity, and survivorship time since diagnosis did not statistically significantly contribute to changes in outcome in this model.

\section{Discussion and Conclusions}

In two recent large population-based studies, statistically significantly higher odds of 1.24 (Canada) and 2.05 (Atlantic Canada) were identified for depressive symptoms among men with a lifetime history of prostate cancer, compared to men without a lifetime history of prostate cancer $[18,19]$. This work extends the current literature by establishing that prostate cancer survivors had 10.23 times $\left(\mathrm{OR}_{\mathrm{MI}}=6.86\right.$ times $)$ higher odds of depressive symptoms than survivors of other types of cancer (other than prostate and skin cancer). To our knowledge, this is the first large population-based study to examine differences between these two groups. It is also the first study to compare depressive symptoms between skin cancer survivors and survivors of other types of cancer. The results point to higher odds of depressive symptoms among male skin cancer survivors compared to other male cancer survivors. However, pooled MI iterations addressing missing data in the outcome variable produced inconclusive results $\left(\mathrm{OR}_{\mathrm{MI}}=2.35, \mathrm{~ns}\right)$.

Coupled with the high incidence and survival rates, numerous factors have been identified as playing a particular role in the mental health outcomes experienced by men with a history of prostate cancer compared to other types of cancer, including physical symptoms (e.g., urinary incontinence), treatment side effects (e.g., erectile dysfunction), anxiety related to potential reoccurrence, and perceptions of masculinity $[3,18]$. The current study highlights the importance of the role of household income and mental health among cancer survivors.
It is well established that household income affects health outcomes, but less attention has been paid to the role of income on mental health - in particular, among cancer survivors [11]. Short and Mallonee [9] established that there are income-related disparities in the quality of life among cancer survivors; those with higher incomes are more likely to have better cancer outcomes, but also experience better quality of life, than those with lower incomes. Mental health is a critical component of healthrelated quality of life for cancer survivors, and the role of household income must be considered in survivorship care and research $[10,11]$.

Low levels of household income are associated with an increased risk of detrimental mental health outcomes $[29,30]$. We have found that this is further compounded among cancer survivors, supporting the limited research among specific groups of cancer survivors, including colorectal cancer and breast cancer survivors $[7,10,11,29,31]$. Our specific focus on prostate cancer survivors further contributes to work highlighting the vulnerability of this population $[3,18,19]$ by demonstrating an increased likelihood of depression compared to men with other forms of cancer and healthy controls, and that this association is moderated by household income. The men with a history of prostate cancer in this sample had more than double the odds of depression than men with any other form of cancer. Furthermore, men with lower annual household incomes (CAD <50,000 and CAD 50,000-99,000) had 2.21 and 1.70 times higher odds of depression, respectively, than those with higher income levels. Research conducted on breast, colorectal and head and neck cancer survivors indicates that low income contributes to lower participation in social events or engagement in social support activities, which may exacerbate feelings of isolation, leading to depression [3235]. The results of the current study address the paucity of data on men's survivorship and mental health research that can help in better understanding these associations and informing survivorship plans with evidence-based findings to prevent their negative effects.

Our findings are also concordant with those that highlight the impact of a prostate cancer diagnosis on both younger $(<65$ years) and unpartnered men $[3,36]$. The younger men in our sample had 2.13 times higher odds of depression than the older men, and the unpartnered men had 1.63 times higher odds. Matheson et al. [36] found that both younger and unpartnered men experience biographical disruption, a sense of marginalization, isolation, and stigma. Younger men, in particular, may experi- 
ence anxiety and/or depression related to incontinence and sexual dysfunction, as compared to older men, who may consider the side effects as a consequence of aging. Similarly, the authors found that unpartnered men reported poorer quality of life and survival outcomes. Unpartnered men may rely on individual and non-partner sources of support and may experience feelings of insecurity related to body image, identity, infertility, and masculinity compared to men with partners. Unpartnered men may also be concerned about disclosure and sexual function in future relationships [36].

This work also serves to highlight the role of multimorbidity in depressive symptoms [37]. In our population, the men with a history of prostate cancer and one or two or more comorbidities had 1.62 and 2.63 times higher odds of depression, respectively, than those with no comorbidities. This finding poses a particular concern in areas such as Atlantic Canada, which has the highest rates of cancer, other chronic diseases, and multimorbidity in the country $[16,21,37-39]$. It is also especially relevant to men with a history of prostate cancer, who have high survival rates and are more likely to develop comorbidities as they age [3].

Research evidence suggests stressful life events can contribute to the onset of depression by means of dysregulating the immune response and disturbing the sensitive interplay among these systems [40]. Surgical and/or radiation therapy complications, together with hormonal therapy, pose significant challenges to patients and may contribute, by means of the stress response they elicit, to the onset of mental distress and depression [41]. Stressors such as urinary incontinence, erectile disfunction (total or partial), and worries about sexual function and intimacy have also been shown to increase mental distress among patients and survivors of prostate cancer [42, 43]. Future studies should consider investigating the role of stress and the negative emotions they elicit in the relationship between prostate cancer survivorship and the development of depression, quality of life, and possible downstream immunological influences on survival rates.

In this study, ethnicity did not appear to play a significant role in our observed outcome. However, other studies have found that men with a current prostate cancer diagnosis with a Hispanic ethnicity and a low household income reported poor mental health [44] and highlighted the role of ethnicity and household income in pre- and post-treatment disparities in urinary, sexual and bowel quality of life [45]. These results may reflect the lack of ethnic diversity in our sample and the geographic area from which our sample is derived (few Hispanics are liv-

Depression and Cancer Survivorship ing in Canada's Atlantic Provinces). The majority of the Atlantic Canada population is White (95\%, of British and Irish decent and $2 \%$ of Frech decent), with the remaining population made up of First Nations and other ethnic groups (3\%) [21].

Research approaches have assumed that men's mental health can be explained in the same terms as women's, or can be assessed and estimated using the same measures. For instance, oncology conferences often lack a presentation or focus section on men's mental health or quality of life. The mental health of men is often overlooked due to inadequate survey assessments of men's experiences and clinicians' subconscious tendency to overlook or not actively seek to assess male distress. This has led to underestimates of the prevalence of depression and anxiety among men. Interdisciplinary and comprehensive investigations and evidence of depression among men with a lifetime history of prostate cancer are only now emerging and beginning to be recognized. This highlights the importance of applying a sex- and gender-based analysis to men's health and mental health research. Recent studies have demonstrated how essential it is to also focus on men's health exclusively, just as they focus on women's health, and that there is a scientific justification for this need $[18,19,38]$.

Our participants with a history of skin cancer had similar odds of depressive symptoms to those with other forms of cancer. We anticipated this, given the high survival rates and the inclusion of both melanoma and nonmelanoma cases in our sample. Bath-Hextall et al. [46] found that although non-melanoma skin cancer patients experienced higher anxiety with a diagnosis than melanoma skin cancer patients, they were not more depressed or anxious than the overall population, and their quality of life improved over a period of 1 year after diagnosis. In this study, the odds of depressive symptoms were statistically significantly higher for skin cancer survivors with a low income than for survivors of other types of cancer with a high income, although in the MI simulation model, the effect disappeared, potentially weakening the validity of the original results for this group. There is limited research in this area, but some of it indicates that non-melanoma skin cancers are associated with heightened levels of distress associated with body image, facial and skin disfigurement from scarring, and unmet needs for care [47-51]. Our findings are in line with those that demonstrate a "small but appreciable" decrement in quality of life related to non-melanoma skin cancer [47]. Furthermore, there are similar findings among long-term melanoma survivors to indicate that there is a slightly in- 
creased risk of depression compared to the general population, but no detrimental impact on global quality of life [52]. Future studies should attempt to replicate the assessment of the association between the presence or absence of skin cancer lifetime diagnosis and the presence of depressive symptoms with a large (preferably complete) data set.

\section{Study Limitations}

This study benefitted from the inclusion of participants from the four Atlantic Canada provinces, where there are high rates of cancer and chronic disease and an aging population. It also benefitted from the inclusion of other relevant sociodemographic factors such as household income, as well as a validated scale to measure depression. However, this study is not without limitations. It is a cross-sectional study, which precludes causal inferences. Future research should attempt to investigate the pathways of influence between social economic status and mental health outcomes on the lifetime trajectory of cancer survivorship before and after diagnosis. The study did not account for patients' time to diagnosis, stage of disease, treatment utilization, or oncological outcomes, which could have impacted the outcome of interest of the current study and deserves future consideration and study. The participant sample was voluntary, and the findings may not be generalizable to cancer survivors from different demographic backgrounds and geographic locations. The data are self-reported and may therefore be subject to bias. Not being able to distinguish between melanoma and nonmelanoma skin cancer cases in the data set limited our interpretation of the results. However, the participants in this cross-sectional cohort will be followed for a period of 30 years, and both the follow-up questionnaire data and future links with administrative health data will allow for this clarification, as well as the identification of incident cancer cases.

The quality of cancer survivorship for men with a history of prostate cancer is critical when considering both the high survival rates and the treatment-related side effects. Mental health as a component of well-being continues to receive less attention in a population that is already less likely to report anxiety and depression. This work demonstrates the role of household income on mental health, in addition to the functional, psychological and social issues experienced by men with a history of prostate cancer, and further supports the need for mental health supports throughout cancer survivorship [53].

\section{Acknowledgments}

We would like to thank the Atlantic PATH participants who donated their time, personal health history, and biological samples to this project. We would also like to thank the Atlantic PATH team members for data collection and management. The team acknowledges the Beatrice Hunter Cancer Research Institute and the Dalhousie Medical Research Foundation (DMRF) Soillse Research Fund for their support.

\section{Statement of Ethics}

This research was conducted ethically in accordance with the World Medical Association Declaration of Helsinki guidelines for human studies and was approved by the Dalhousie University Research Ethics Board (Project No. 2018-4462). All participants had provided written informed consent prior to participating in the study.

\section{Conflict of Interest Statement}

The authors declare that the research was conducted in the absence of any commercial or financial relationships that could be construed as a potential conflict of interest.

\section{Funding Sources}

The data used in this research were made available by the Atlantic PATH study, which is the Atlantic Canada regional component of the Canadian Partnership for Tomorrow's Health funded by the Canadian Partnership against Cancer and Health Canada. The views expressed herein represent the views of the authors and do not necessarily represent the views of Health Canada. Data access was funded by the Beatrice Hunter Cancer Research Institute (March 2020) and Dalhousie Medical Research Foundation (DMRF) Soillse Research Fund.

\section{Author Contributions}

All authors contributed to the development of the hypotheses and objectives of the study; E.S. prepared the data set for analysis; G.I. conducted the statistical analyses in consultation with E.S. and R.R.; all authors contributed to the drafting of the manuscript and subsequent revisions. 


\section{References}

1 Naughton MJ, Weaver KE. Physical and mental health among cancer survivors: considerations for long-term care and quality of life. $\mathrm{N}$ C Med J. 2014;75(4):283-6.

2 DiMatteo MR, Lepper HS, Croghan TW. Depression is a risk factor for noncompliance with medical treatment: meta-analysis of the effects of anxiety and depression on patient adherence. Arch Intern Med. 2000;160(14): 2101-7.

3 Fervaha G, Izard JP, Tripp DA, Rajan S, Leong DP, Siemens DR. Depression and prostate cancer: a focused review for the clinician. Urol Oncol. 2019;37(4):282-8.

4 Linden W, Vodermaier A, Mackenzie R, Greig D. Anxiety and depression after cancer diagnosis: prevalence rates by cancer type, gender, and age. J Affect Disord. 2012;141(23):343-51.

5 Adler NE, Page AEK; Institute of Medicine (US). Cancer care for the whole patient: meeting psychosocial health needs. Washington (DC): National Academies Press (US); 2008.

6 Ekwueme DU, Lunsford NB, Khushalani JS, Rim SH. Public health efforts to address mental health conditions among cancer survivors. Am J Public Health. 2019 Jun;109(S3):S17980.

7 Lundy JJ, Coons SJ, Wendel C, Hornbrook MC, Herrinton L, Grant M, et al. Exploring household income as a predictor of psychological well-being among long-term colorectal cancer survivors. Qual Life Res. 2009; 18(2):157-61.

8 Roick J, Danker H, Kersting A, Dietrich A, Dietz A, Papsdorf K, et al. The association of socioeconomic status with quality of life in cancer patients over a 6-month period using individual growth models. Support Care Cancer. 2019;27(9):3347-55.

9 Short PF, Mallonee EL. Income disparities in the quality of life of cancer survivors. Med Care. 2006;44(1):16-23.

10 Alawadi SA, Ohaeri JU. Health-related quality of life of Kuwaiti women with breast cancer: a comparative study using the EORTC Quality of Life Questionnaire. BMC Cancer. 2009;9:222.

11 Nayak MG, George A, Vidyasagar MS, Mathew S, Nayak S, Nayak BS, et al. Quality of life among cancer patients. Indian J Palliat Care. 2017 Oct-Dec;23(4):445-50.

12 Canadian Cancer Society [Internet]. Melanoma: deadliest type of skin cancer is on the rise [cited 2020 Jun 15]. Available from: https:// www.cancer.ca/en/about-us/for-media/media-releases/national/2014/2014-canadiancancer-statistics/?region $=$ on .

13 Canadian Cancer Society [Internet]. Survival statistics for non-melanoma skin cancer [cited 2020 Jun 15]. Available from: https://www. cancer.ca/en/cancer-information/cancertype/skin-non-melanoma/prognosis-andsurvival/survival-statistics/?region $=$ on .
14 Brenner DR, Weir HK, Demers AA, Ellison LF, Louzado C, Shaw A, et al. Projected estimates of cancer in Canada in 2020. CMAJ. 2020;192(9):E199.

15 Canadian Cancer Statistics Advisory Committee [Internet]. Canadian Cancer Statistics 2019. Toronto, ON: Canadian Cancer Society; 2019 [cited 2019 Jun]. Available from: www.cancer.ca/Canadian-Cancer-Statistics2019-EN.

16 Canadian Cancer Society [Internet]. Canadian statistics at a glance [cited 2020 Jun]. Available from: https://www.cancer.ca/en/cancerinformation/cancer-101/cancer-statistics-ata-glance/?region $=$ on .

17 Canadian Cancer Society [Internet]. Special topic: Predictions of the future burden of cancer in Canada [cited 2020 Jun]. Available from: https://www.cancer.ca/ /media/cancer.ca/CW/cancer\%20information/cancer\%20101/Canadian\%20cancer\%20statistics/Canadian-Cancer-Statistics-2015-EN. pdf.

18 Ilie G, Rutledge R, Sweeney E. Anxiety and depression symptoms in adult males in Atlantic Canada with or without a lifetime history of prostate cancer. Psychooncology. 2020 Jun; 29(2):280-6.

19 Moodie L, Ilie G, Rutledge DHR, Panteleo A, Kirkland S. Current mental health status among a population-based sample of Canadian men with and without a history of prostate cancer diagnosis. Front Psychiatry. 2020 [Submitted, currently under review].

20 Watts S, Leydon G, Birch B, Prescott P, Lai L, Eardley S, et al. Depression and anxiety in prostate cancer: a systematic review and meta-analysis of prevalence rates. BMJ Open. 2014 Jan;4(3):e003901.

21 Sweeney E, Cui Y, DeClercq V, Devichand P, Forbes C, Grandy S, et al. Cohort profile: the Atlantic Partnership for Tomorrow's Health (Atlantic PATH) study. Intern J Epidem. 2017 Dec;46(6):1762-3.

22 Dummer TJB, Awadalla P, Boileau C, Craig C, Fortier I, Goel V, et al. The Canadian Partnership for Tomorrow project: a pan-Canadian platform for chronic disease prevention research. CMAJ. 2018 Jun;190(23):E710-7.

23 Yu ZM, Parker L, Dummer TJB. Depressive symptoms, diet quality, physical activity, and body composition among populations in Nova Scotia, Canada: report from the Atlantic Partnership for Tomorrow's Health. Prev Med. 2014 Apr;61:106-13.

24 Kroenke K, Spitzer RL, Williams JB. The PHQ-9: validity of a brief depression severity measure. J Gen Intern Med. 2001 Sep;16(9): 606-13.

25 Lowe B, Kroenke K, Herzoga W, Grafe K. Measuring depression outcome with a brief self-report instrument: sensitivity to change of the Patient Health Questionnaire (PHQ-9). J Affect Disord. 2004;81(1):61-6.
26 Martin A, Rief W, Klaiberg A, Braehler E. Validity of the brief Patient Health Questionnaire mood scale (PHQ-9) in the general population. Gen Hosp Psychiatry. 2006 Jan-Feb; 28(1):71-7.

27 Sterne JA, White IR, Carlin JB, Spratt M, Royston P, Kenward MG, et al. Multiple imputation for missing data in epidemiological and clinical research: potential and pitfalls. BMJ. 2009 Jun;338:b2393.

28 van Buuren S. Flexible imputation of missing data. CRC Press, Taylor and Francis Group; 2018.

29 Seedat S, Scott KM, Angermeyer MC, Berglund P, Bromet EJ, Brugha TS, et al. Crossnational associations between gender and mental disorders in the World Health Organization world mental health surveys. Arch Gen Psychiatry. 2009 Jul;66(7):785-95.

30 Sareen J, Afifi TO, McMillan KA, Asmundson GJ. Relationship between household income and mental disorders: findings from a population-based longitudinal study. Arch Gen Psychiatry. 2011 Apr;68(4):419-27.

31 Ashing-Giwa KT, Lim JW. Examining the impact of socioeconomic status and socioecologic stress on physical and mental health quality of life among breast cancer survivors. Oncol Nurs Forum. 2009 Jan;36(1):79-88.

32 Gandhi AK, Roy S, Thakar A, Sharma A, Mohanti BK. Symptom burden and quality of life in advanced head and neck cancer patients: AIIMS study of 100 patients. Indian J Palliat Care. 2014 Sep;20(3):189-93.

33 Maughan TS, James RD, Kerr DJ, Ledermann JA, McArdle C, Seymour MT, et al. Comparison of survival, palliation, and quality of life with three chemotherapy regimens in metastatic colorectal cancer: a multicentre randomised trial. Lancet. 2002 May;359(9317): 1555-63.

34 Knobf MT, Thompson AS, Fennie K, Erdos $D$. The effect of a community-based exercise intervention on symptoms and quality of life. Cancer Nurs. 2014 Mar-Apr;37(2):E43-50.

35 Wyatt G, Sikorskii A, Tamkus D, You M. Quality of life among advanced breast cancer patients with and without distant metastasis. Eur J Cancer Care (Engl). 2013 Mar;22(2): 272-80.

36 Matheson L, Watson EK, Nayoan J, Wagland $\mathrm{R}$, Glaser A, Gavin A, et al. A qualitative metasynthesis exploring the impact of prostate cancer and its management on younger, unpartnered and gay men. Eur J Cancer Care (Engl). 2017 Nov;26(6).

37 Knoll AD, MacLennan RN. Prevalence and correlates of depression in Canada: findings from the Canadian Community Health Survey. Can Psychol. 2017;58(2):116-23.

38 Smith DT, Mouzon DM, Elliott M. Reviewing the assumptions about men's mental health: an exploration of the gender binary. Am J Mens Health. 2018 Jan;12(1):78-89. 
39 Keats MR, Cui Y, DeClercq V, Dummer TJB, Forbes C, Grandy SA, et al. Multimorbidity in Atlantic Canada and association with low levels of physical activity. Prev Med. 2017;105: 326-31.

40 Pinto A, Faiz O, Davis R, Almoudaris A, Vincent C. Surgical complications and their impact on patients' psychosocial well-being: a systematic review and meta-analysis. BMJ Open. 2016;6:e007224.

41 Kong EH, Deatrick JA, Bradway CK. Men's experiences after prostatectomy: a meta-synthesis. Int J Nurs Stud. 2017;74:162-71.

42 Ilie G, White J, Mason R, Rendon R, Bailly G, Lawen J, et al. Current mental distress among men with a history of radical prostatectomy and related adverse correlates. Am J Mens Health. 2020 Sep-Oct;14(5):1557988320957535.

43 Ilie G, MacDonald M, Bell D, Rendon R, Langille G, Bailly G, et al. An examination of the relationship between mental distress, functional and psychosocial quality of life indicators in a population-based sample of prostate cancer survivors who received curative treatment. Urol Pract. 2020;23(2):10-5.
44 Gore JL, Krupski T, Kwan L, Maliski S, Litwin MS. Partnership status influences quality of life in low-income, uninsured men with prostate cancer. Cancer. 2005 Oct;104(1):191-8.

45 Orom H, Biddle C, Underwood W 3rd, Homish GG, Olsson CA. Racial or ethnic and socioeconomic disparities in prostate cancer survivors' prostate-specific quality of life. Urology. 2018 Feb;112:132-7.

46 Bath-Hextall F, Jenkinson C, Kumar A, Leonardi-Bee J, Perkins W, Cox K, et al. Longitudinal, mixed method study to look at the experiences and knowledge of non melanoma skin cancer from diagnosis to one year. BMC Dermatol. 2013 Oct 29;13(13):13.

47 Gaulin C, Sebaratnam DF, Fernández-Peñas P. Quality of life in non-melanoma skin cancer. Australas J Dermatol. 2015 Feb;56(1):706.

48 Körner A, Garland R, Czajkowska Z, Coroiu A, Khanna M. Supportive care needs and distress in patients with non-melanoma skin cancer: nothing to worry about? Eur J Oncol Nurs. 2016 Feb;20:150-5.
49 Rhee JS, Matthews BA, Neuburg M, Logan BR, Burzynski M, Nattinger AB. Validation of a quality-of-life instrument for patients with nonmelanoma skin cancer. Arch Facial Plast Surg. 2006 Sep;8(5):314-8.

50 Sobanko JF, Sarwer DB, Zvargulis Z, Miller CJ. Importance of physical appearance in patients with skin cancer. Dermatol Surg. 2015 Feb;41(2):183-8.

51 Vinding GR, Christensen KB, Esmann S, Olesen AB, Jemec GB. Quality of life in non-melanoma skin cancer - the Skin Cancer Quality of Life (SCQoL) questionnaire. Dermatol Surg. 2013 Nov;39(12):1784-93.

52 Beutel ME, Fischbeck S, Binder H, Blettner M, Brähler E, Emrich K, et al. Depression, anxiety and quality of life in long-term survivors of malignant melanoma: a register-based cohort study. PLoS One. 2015 Jan;10(1):e0116440.

53 Ilie G, Mason R, Bell S, Bailly G, Rendon RA, Mann R, et al. Development and initial evaluation of a multifaceted intervention to improve mental health and quality of life among prostate cancer survivors. Int J Ment Health Addict. 2019;26:1-14. 